Lorenzo E. Guglielmi, Aleksandra R. Blatešić

loreguglie@yahoo.it; lorenzo.guglielmi@unive.it

(Università Ca' Foscari di Venezia)

ablatesic@yahoo.it

(Filozofski fakultet - Romanistika: Italijanski jezik, Novi Sad)

\title{
APPRENDERE LE MICROLINGUE IN TELETANDEM, TRA TEORIA E PRATICA
}

\section{ABSTRACT}

Nel Teletandem (TT) due apprendenti comunicano in ambiente telematico per imparare l'uno la lingua madre dell'altro, in modo autonomo e secondo un progetto personalizzato di scambio linguistico-culturale. Nel caso di un TT con le microlingue, inoltre, è possibile configurare un rapporto di accentuata simmetria tra addetti di uno stesso settore scientifico-professionale e il superamento del modello che prevede la relazione tra un insegnante di microlingua, in genere non specialista della materia, possessore del significante, $\mathrm{e}$ un discente, possessore dei significati. Il presente lavoro intende illustrare le criticità e i punti di forza nell'ambiente di apprendimento TT quando in esso entrano in gioco i linguaggi settoriali.

PAROLE CHIAVE: apprendimento di microlingue, Teletandem, autonomia dell'apprendente, counseling, approccio task-based.

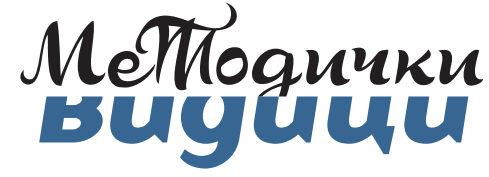




\section{Le microlingue e l'apprendimento in ambiente telematico}

econdo la definizione di Balboni, le microlingue scientifico-professionali sono usate "con lo scopo di una comunicazione che sia il meno ambigua possibile e del riconoscimento di appartenenza ad un settore scientifico-professionale" (Balboni 2000, 21-22). ${ }^{1)}$ Le microlingue dunque presentano aspetti morfosintattici, lessicali e testuali nettamente distinguibili, sia nel senso di langue che in quello di parole. La comunicazione viva in campo scientifico e professionale presenta di frequente un'osmosi tra diversi registri mentre la conoscenza della langue nel contesto specifico della comunicazione microlinguistica permette di disambiguare e condividere i messaggi tra specialisti di uno stesso settore scientifico-professionale.

L'apprendimento-insegnamento di una microlingua si caratterizza in genere come un rapporto nel quale il discente, specialista in un determinato settore, si affida alla mediazione di un esperto linguistico che lo facilita nell'apprendere a ricodificare nella lingua obiettivo i contenuti della materia già espressi in una o più microlingue di partenza (siano esse LM, LS1, LS2, ecc.). Questo tipo di apprendimento gli consentirà di partecipare, a seconda del grado di raggiunto nello sviluppo delle competenze comunicative, alla comunità scientifico-professionale che di quella microlingua fa uso, in una serie di possibili contesti comunicativi caratterizzati da compiti scritti e/o orali, conferenza, seminario, scrittura di saggio, ecc. A meno che l'esperto linguistico in questione - in materia di economia, biologia, matematica, ecc. - non sia anch'egli uno specialista nella materia veicolata, il raggiungimento di un certo livello di proficiency nella microlingua obiettivo sarà possibile per lo più affidandosi alla capacità collaborativa tra il discente (esperto della materia), possessore del significato, e il docente (esperto linguistico), possessore del significante. Tale proficiency sarà l'interfaccia microlinguistica che renderà comunicabili i contenuti della materia veicolata all'interno della comunità.

Questo modello operativo della lezione di microlingua potrebbe tuttavia essere superato qualora fossero i

1) L'argomento trattato in questo articolo è stato presentato al II Convegno Internazionale "Language for specific purposes: Challenges and prospects", a Belgrado, 05 febbraio 2011. due esperti dello stesso settore scientifico-professionale ad imparare ciascuno la (micro)lingua (madre) dell'altro. Con l'uso delle parentesi intendiamo evidenziare come in un sistema di apprendimento microlinguistico di tipo tandem, sia esso in presenza o a distanza, l'elemento "micro", seppur necessario, non eserciti un dominio assoluto, mentre l'elemento "madre" non sia la conditio sine qua non. È, infatti, possibile che due esperti di uno stesso settore scientifico professionale si forniscano reciprocamente sostegno nell'apprendere ciascuno dall'altro la microlingua obiettivo nel quale hanno un alto livello di proficiency come LS1, LS2, LS3, ecc. In questo rapporto didattico, inoltre, saranno presenti e si svilupperanno obiettivi di apprendimento ed effetti di feedback su altre componenti della competenza comunicativa non direttamente riconducibili alla microlingua.

Nel caso in cui le lingue (eventualmente LM) conosciute dai partecipanti siano al contempo (micro)lingue obiettivo del rapporto di tandem, si configura tuttavia uno spazio didattico-comunicativo che, a differenza del contesto classico della didattica della microlingue, possiede le seguenti caratteristiche:

- il bilinguismo, individualmente asimmetrico, nell'interazione tra due soggetti in tandem si attiva in modo speculare;

- entrambi i soggetti, essendo esperti della materia veicolata, possiedono il significato oltre al significante della microlingua di cui sono esperti.

Il nostro contributo prende in esame le modalità di apprendimento delle microlingue della letteratura e della linguistica italiana e serba nel Teletandem (TT), da intendersi come strumento di studio a distanza che prevede un alto livello di autogestione, autonomia e personalizzazione (cf. Brammerts, 2003). Si tratta di una modalità basata sull'apprendimento autonomo di una LS che consente agli studiosi di un determinato settore di incontrarsi on line per imparare l'uno la (micro)lingua dell'altro. Nel nostro caso si tratta di una sperimentazione basata su una coppia avente il seguente profilo:

- L., madrelingua italiana con laurea in lingua e letteratura serba di secondo livello presso l'Università di Torino, traduttrice. 
- J., madrelingua serba ${ }^{2)}$, laureata in studi magistrali presso la Facoltà di Filosofia di Novi Sad, Dipartimento di Romanistica (francese LS1, italiano LS2).

Questo progetto TT, che andremo ad illustrare in alcuni suoi aspetti, è basato in parte sui principi dell'originale Teletandem denominato Teletandem Brasil: Foreign Languages for $\mathrm{All}^{3)}$, creato nel 2006 da un gruppo di ricercatori brasiliani con lo scopo di dare la possibilità agli studenti universitari di lingue straniere di sviluppare contemporaneamente le capacità linguistico-formative e didattiche a distanza impiegando dei software come Windows Live Messenger, Skype, ooVo ecc. Qui riportiamo i sei aspetti chiave dell'originale progetto, chiaramente diverso e decisamente più complesso di un semplice chatting (Telles, Vassallo, 2006: 194):

1. L'uso di computer e nuove tecnologie consente l'apprendimento di una LS2 a distanza attraverso la produzione scritta e orale, lettura, ascolto, comprensione e webcam.

2. A partire dal protocollo condiviso l'ambiente TT influisce sulla costruzione di un rapporto di reciprocità e autonomia tra i partecipanti.

3. I partecipanti al progetto sono interessati all'imprendimento della lingua madre del partner.

4. I partecipanti possiedono competenze linguistiche nelle rispettive lingue ma non devono essere parlanti nativi né insegnanti professionisti.

5. Le sessioni nel TT si svolgono regolarmente e potenziano le capacità (meta)linguistiche e didattiche.

6. Le conversazioni e le produzioni scritte sono archiviate e permettono revisione e (auto)correzione del materiale prodotto.

Dal momento che la nostra ricerca è orientata all'analisi dell'apprendimento di microlingue abbiamo dovuto impostare il progetto modificando alcuni aspetti dell'originale Teletandem. Allo stato attuale, sono coinvolti due partner parlanti nativi insegnanti-ricercatori di una stessa

\footnotetext{
2) Da questo momento utilizzeremo le denominazioni normalmente utilizzate nel mondo anglosassone, NS=madrelingua e NNS=non madrelingua, con riferimento alle interazioni di TT.

3) Per ulteriori informazioni consultare il sito: www.teletandembrasil.org
}

area di studi umanistici. Ciò implica che all'atto didattico e di apprendimento del TT partecipano due soggetti il cui legame di interdipendenza è fondato su un'affinitàcomplementarietà delle competenze e su un simile livello di responsabilità. D'altra parte, l'inserimento dell'attività di tutoraggio al progetto TT si impone come un passo pressoché obbligato, poiché la verifica dei livelli di partenza, le modalità di comunicazione e formazione delle coppie, la somministrazione della piattaforma on line, il raggiungimento degli obiettivi ex post ed analisi dei risultati sono solo alcuni aspetti di una complessa modalità di apprendimento che richiedono la mediazione di uno o più tutor esterni, ossia di counselors.

Il semplice fatto che un TT microlinguistico apra all'eventualità di mettere in contatto on line e far conoscere per la prima volta due specialisti di uno stesso settore e che questi possano anche non aver alcun tipo di esperienza pregressa con la microlingua, di per sé, può già giustificare la presenza di uno "sguardo esterno", cioè di uno o più counselors impegnati in un'azione di scaffolding e monitoraggio dell'ambiente. Questa serie di riflessioni riconducono il discorso alla fonte del tandem, ossia al concetto di autonomia.

\section{Concetti base dell'apprendimento cooperativo nel TT: autonomia, counseling e approccio task-based}

Come si è visto nel precedente paragrafo nelle sessioni TT è consentito l'archivio degli scambi scritti e orali, il che offre l'opportunità di rivedere e ripensare le produzioni realizzate tra le coppie. Alcuni ricercatori come David Little ritengono che la riflessione sia uno dei fattori più importanti nel favorire lo sviluppo dell'autonomia: "Reflection plays a central role in the development and exercise of learner autonomy; and writing things down plays a central role in developing our capacity for reflection" (Little 2002: 27). Cosa sia l'autonomia resta una questione, tuttavia, ancora molto aperta, ma qualsiasi sperimentazione di modelli operativi nel Teletandem non può prescindere dalla riflessione su questo concetto.

Secondo l'interpretazione di Holec, l'autonomia è "capacità di farsi carico del proprio apprendimento" (Ho- 
lec, 1981: 3), ma tale capacità, sempre secondo Little, non può che generarsi nell'interazione, perché non esiste alcuna indipendenza se non a partire da una base di interdipendenza (White, 2007: 61). L' interdipendenza positiva, all'interno di un rapporto che Varisco definisce come "interscambio dialettico" (Dolci, 2004: 84) per raggiungere una "costruzione di con-senso", significa reciproco affidamento dei partners: gli apprendenti di microlingua in TT devono sentire tanta responsabilità per l'apprendimento del partner quanto quella che sentono per il proprio apprendimento. È presumibile che due studenti di microlingua possiedano già un grado individuale di autonomia a cui affidarsi. La solidità e l'ampiezza della loro base di autonomia, in senso generale, tenderanno ad essere in un rapporto di proporzionalità diretta con il numero di LS conosciute e con il grado di esperienza pregressa nell'apprendimento microlinguistico, compo-

nenti che giocano un ruolo primario nello sviluppo della competenza glottomatetica (cf. Balboni, 2002). Se da un lato tutte queste componenti facilitano l'affinamento di strategie di transfer of training oltre che di positive transfer (cf. Odlin, 1989; Cenoz, Hufeisen \& Jessner, 2001; Cenoz, 2001) tra le microlingue già possedute (LM, LS1, LS2, ecc.) e la microlingua obiettivo, dall'altro, è pur vero che in un ambiente di apprendimento microlinguistico di collaborazione tra pari sono due le autonomie ad essere messe in circolo e dunque sfidate. La sfida consiste nelle abilità sociali richieste dal tipo di ambiente di apprendimento e nel carico psicologico ed emotivo che ne deriva. La consapevolezza che il TT sia innanzi tutto una modalità collaborativa di studio ci porta a fare riferimento alla definizione di Anderson e Garrison, i quali considerano l'autonomia nell'apprendimento a distanza come learner's control (White, 2007: 60-61).

Attraverso il seguente grafico (cf. Anderson\&Garrison, fonte: White, 2007: 60-61), da noi elaborato, abbiamo pre-
FIGURE 1

In(ter)dipendenza nell' apprendimento a distanza

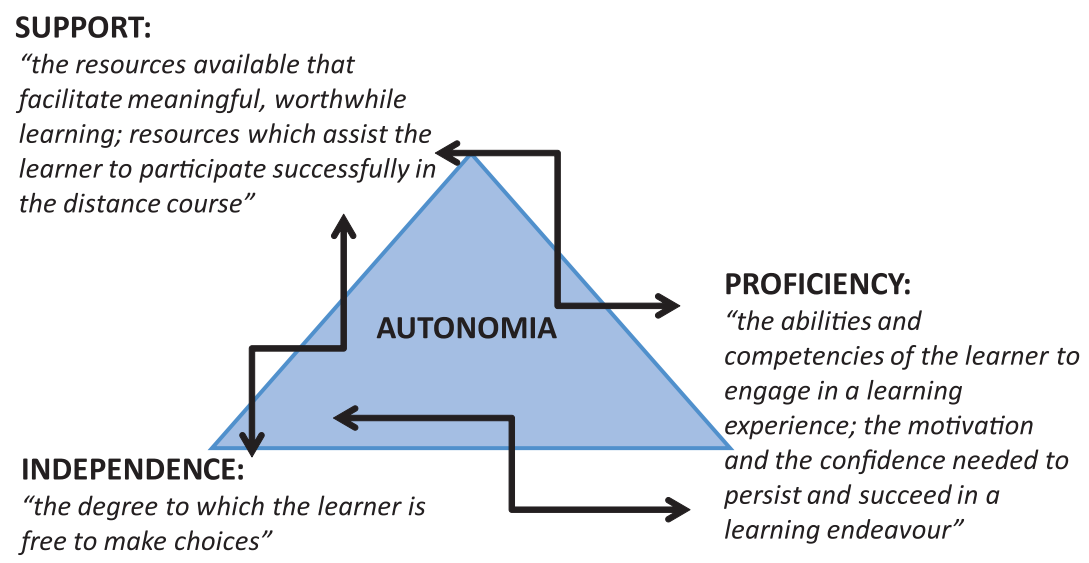

Adattato da Anderson\&Garrison, fonte: White, 2007

sentato i tre fattori fondamentali per lo sviluppo dell'autonomia nel TT.

L'organizzazione del counseling, dunque, può costituire una risorsa valida per la realizzazione di una comunicazione efficace all'interno della coppia, per lo sviluppo dell'autonomia e il sostegno dei fattori motivazionali. Gli obiettivi dichiarati e le istruzioni dovrebbero fornire agli studenti solo uno scaffolding all'interno del quale conversare liberamente esercitando le proprie abilità di problem-solving (cf. Mullen et al., 2009), tanto in senso linguistico (alternando focus on meaning and focus on form), quanto nell'organizzazione e nell'interpretazione del proprio spazio di lavoro.

Forniamo di seguito un esempio del programma task-based presente nella piattaforma wiki $P B$ works, ${ }^{4)}$ sulla quale stiamo attualmente svolgendo la nostra prima sperimentazione di TT con microlingue.

4) Per creare una piattaforma wiki PBworks: http://pbworks.com 


\section{TAB. A PROGRAMMA TASKS TELETANDEM E MICROLINGUE}

I settimana TT: avete inziato a conoscervi e a sperimentare l'interazione in TT (protocollo di base). Se avete domande a riguardo o problemi che possiamo aiutarvi a risolvere, non esitate a chiedere sul FORUM

I FASE Teletandem microlinguistico "task based"

\section{TASK:}

1. Caricate i rispettivi CV e un estratto (un capitolo) delle vostre tesi oppure degli articoli o saggi che avete scritto, in qualsiasi lingua, dentro i folders J._lavori in corso e L._lavori in corso (folder Tasks in progress).

2. Fate una prima lettura di tutta la documentazione.

3. Ricavate delle domande per un'intervista al partner con focus sulle sue caratteristiche caratteriali da abbinare a un'indagine il suo percorso di apprendimento delle lingue e delle culture straniere e sulle esperienze professionali e di vita.

4. Concedetevi di non prendervi troppo sul serio! L'importante è reimpiegare ed esplorare quei campi di lessico che riguardano il settore scientifico e professionale.

5. Intervista via Skype: 1 ora in serbo/1 ora in italiano: (chat e videochiamata)

6. Rileggete e riascoltate le tracce delle interviste e ricavate un profilo del partner in III persona da caricare in L._lavori in corso o J._lavori in corso.

\section{TASK: AVETE UN POSSIBILE TOPIC COMUNE?}

1. Fate una lettura critica degli estratti di tesi e/o degli articoli o dei saggi che vi siete caricate sulla piattaforma. Cercate possibili connessioni anche a livello interdisciplinare tra i vostri scritti (Lingua $>$ Letteratura /Letteratura $>$ Lingua).

2. Sottolineate tutte le frasi, le parole o i contenuti che non vi sono chiari.

3. Preparate una serie di domande per il partner relative ai contenuti dei suoi scritti.

4. Cercate di capire se c'è una o più possibilità di sviluppare un topic comune, confrontandovi, anche più in generale, sui vostri possibili interessi di ricerca, sulle altre vostre pubblicazioni in cantiere o già completate.

5. Discutetene via Skype 45 minuti in serbo partendo dagli scritti di J. e 45 minuti in italiano traendo spunto dagli scritti di L.. Aiutatevi a chiarirvi a vicenda tutto ciò che non è chiaro per ragioni di lingua e/o contenuti.

Obiettivi linguistici: ripasso, rinforzo, reimpiego del linguaggio di settore con confronto dialettico.

Obiettivi formativi generali: confrontarsi sulle prossime fasi del progetto

II fase, percorso A: Se state trovando un topic comune, allora potete provare ad organizzare un articolo o un saggio a due mani, che verrà pubblicato in versione bilingue italiano-serbo.

II fase, percorso B: Se non riuscite a trovare un topic comune, allora vi aiuterete reciprocamente a riflettere su come potreste sviluppare due lavori individuali 
Avete trovato il Topic. Esempio: „Il linguaggio pubblicitario in serbo e in italiano"

Ora dovete decidere come sviluppare il tema: quali aspetti prendereste in esame? Avete già della bibliografia? C'è già della letteratura sul tema?

- Fate una o più sessioni TT via Skype per discuterne.

- Usate il FORUM per scambiarvi idee con commento.

- A questo punto, ognuna di voi costruisce la traccia di un articolo con alcuni titoli di paragrafi e capitoli, lo carica sulla piattaforma e se lo fa commentare.

- Quindi vi mettete d'accordo su come dividervi le parti e su chi incomincia a scrivere l'introduzione

Costruzione dell'articolo "a staffetta":

Esempio:

\section{I composizione e revisione}

J. scrive l'introduzione in italiano > L. commenta, aggiunge, corregge, trova i punti critici da punto di vista morfosintattico, lessicale, ecc. La correzione deve essere prevalentemente rilevativa, in modo che il partner possa tornare sui propri errori e autocorreggerli> L. prosegue in serbo con primo paragrafo del primo capitolo. J. fa la correzione. E così via fino alla bozza finale.

\section{Seconda revisione con aggiustamenti}

Arrivati alla bozza finale, si passa alla seconda revisione. Ciascuno revisiona la propria parte, autocorreggendo gli errori rilevati dal proprio partner.

\section{Traduzione}

Infine, L. traduce in italiano le parti scritte e revisionate in serbo e J. traduce in serbo le parti scritte e revisionate in italiano. In questo modo arriveremo all'articolo completo in entrambe le lingue, scritto a due mani.

Strumenti da utilizzare: piattaforma Danubadria personale per caricare documenti word, FORUM interno di piattaforma per avvisare il partner e commentare, SKYPE per correzioni in chat e orali sincroniche.

\section{II fase, percorso B: ognuno scrive il proprio lavoro con la consulenza del partner madrelingua}

Ogni partner aiuta l'altro a focalizzarsi sulla ricerca del TOPIC e sullo sviluppo del tema dell'articolo o del saggio, anche suggerendo indicazioni bibliografiche e materiali di approfondimento.

Strumenti da utilizzare: piattaforma Danubadria personale per caricare documenti word, FORUM interno di piattaforma per avvisare il partner e commentare, SKYPE per correzioni in chat e orali sincroniche. 
Il lavoro può essere impostato nel seguente modo:

- ognuno scrive il proprio articolo in lingua madre e poi lo traduce nella LS

- ognuno cerca di scrivere direttamente nella LS

- formula ibrida

Per la revisione vale sempre lo stesso meccanismo: caricare (funzione upload) in L._lavori in corso/J._lavori in corso (cartella Tasks in progress), lasciare avviso su FORUM, download/revisione/nuovo upload, fino alla versione che vi soddisfa.

III FASE:

Presentazione ppt. nelle due lingue del lavoro comune o dei due lavori.

Recensioni nel caso si tratti di due lavori.

- Preparate dei ppt., revisionate con il solito sistema L./J._lavori in corso e usate Skype con videocamera per simulare la presentazione ppt. accademica

Nel caso di due lavori separati (v. percorso B) preparate due recensioni

\section{Analisi delle interazioni in TT con microlingue su chat e su forum wiki}

Dal nostro punto di vista è interessante esaminare il tipo di interazioni tra pari (partners) e tra partners e counselors che si possono generare attraverso l'uso combinato dei canali Skype con una piattaforma wiki Pbworks, nell'ambito delle varie attività comunicative riconducibili al processo di mediazione dei tasks (cf. Guk\&Kellog, 2007).

Il momento dell'interazione in TT che analizzeremo è quella in cui due partners L. e J., rispettivamente di madrelingua italiana e serba, stanno entrando nel vivo del percorso A del programma di lavoro (v.TAB. A), percorso a cui sono giunte insieme, discutendone in entrambe le lingue sulla chat.

Dall'estratto di conversazione in chat (v.TAB. B) emerge chiaramente una tendenza di entrambi i partner a comunicare con lo scopo di negoziare (v. frasi segnate con la siglia $[\mathbf{N}])$ temi, materiali e modalità di lavoro. Questa è la principale funzione di metalinguaggio in gioco. Il numero di errori commessi dal NNS (L.) e non corretti dal NS (J.), sottolineati da noi nell'estratto di chat (v.legenda in TAB. $\mathrm{B})$, sembrerebbe piuttosto alto, ma corrisponde in realtà a una strategia ben precisa nell'azione di sostegno del NS (J.) sul NNS (L.) e nella realizzazione degli obiettivi comuni considerati prioritari. Questo aspetto emergerà in seguito anche sul forum, sotto forma di riflessione esplicita con i counselors (v. TAB. C). È, infatti, l'errore terminologico dato dall'uso della parola član al posto di članak a diventare il focus unico dell'intero processo di correzione. Pur trattandosi di un termine non strettamente accademico, essa riveste un'importanza fondamentale per l'apprendente NNS di microlingua. La sua collocazione nello specifico contesto sintattico e di interazione (clan ko prica o juoslevenski pozoriste (sic) = tradotto letteralmente, "un membro che parla di teatro jugoslavo" anziché un "articolo") rende necessaria un'attività più profonda di disambiguazione (v. frasi segnate con [E.C.] in TAB. B.) Questa attività di correzione dell'errore e di riflessione partecipata si inserisce nel processo dominante di negoziazione sui tasks senza ostacolarlo. Un tale stile di correzione dell'errore rivela lo stretto legame di alternanza e compresenza di focus on form e focus on meaning nel TT.

Nel frammento di chat esaminato solo un banale errore terminologico - riconducibile a un transfer negativo della $\mathrm{LM}^{5)}$ ma ritenuto, a ragione rilevante ai fini di

5) In italiano il sostantivo articolo identifica sia un elemento grammaticale che un'opera scritta, scientifica o giornalistica. 
un'efficace comprensione tra partners in fase negoziale - diventa oggetto di un'accurata correzione da parte del NS. È tuttavia interessante notare come la prima spiegazione fornita dal NS [v. frase contrassegnata dal simbolo (\#)] sia caratterizzata da alcuni errori di battitura potenzialmente rilevanti sul piano di una possibile ricezione distorta del messaggio da parte del NNS. Tutti gli errori sottolineati e non corretti, riguardano per lo più aspetti legati alla flessione nominale e non risultano ostativi all'efficacia comunicativa nel quadro degli obiettivi di lavoro proposti nel programma (cf. TAB. A). Altri errori [v. ${ }^{\circ}{ }^{\circ}$.C. ${ }^{\circ}$ ] vengono invece trattati semplicemente ripetendo le frasi del NNS e incorporandole in forma corretta nel metalinguaggio della negoziazione [N.] sottoforma di feedback.

\section{TAB. B}

Legenda: [N.]: negoziazione/ parole sottolineate nel testo: errore non corretto / in bold*: errore su cui si focalizza la correzione e la riflessione/[E.C.]: processo di correzione dell'errore e di disambiguazione $/{ }^{\circ \circ}[\text { E.C. }]^{\circ \circ}$ : correzione dell'errore senza rilevarlo direttamente, ma ripetendo elementi del discorso del NNS in forma corretta/[F.]: feedback del NNS al NS/[I.]: Inquiring, richiesta di spiegazione /(\#): frase con errore di battitura con possibili effetti distorsivi nella comprensione-interazione

\section{$(\ldots)$}

[2:07:11 PM] J.: nemam ništa novo. gledala sam još časopisa orhestra, ima kritika nekih stranih, prevedenih predstava, ali nisam shvatila zašto misliš da je to važno.

Non ho nulla di nuovo. Ho guardato ancora riviste. Ci sono le critiche di alcune rappresentazioni straniere tradotte, ma non mi è chiaro perchè sia così importante per te. [N. Negoziazione sui contenuti e sui materiali da trattare]

[2:08:06 PM] 1.: Nema problema...pomislila sam da mozemo ${ }^{\circ}$ objiati delovi ${ }^{\circ \circ}$ dokumenta na saitu danubadria. Sta mislis? Non importa...pensavo che si poteva pubblicare delle parti dei documenti sul sito Danubadria [N.]

[2:09:33 PM] J.: verovatno si mislila jer u tekstu o predstavi "Dragomira" spominju i prevod /forse ci hai pensato dal momento che nel testo dell'opera "Dragomira" fanno riferimento a una traduzione [N.]

[2:09:45 PM] J.: važi, možemo ${ }^{\circ}$ objaviti delove [E.C.] ${ }^{\circ}$ prevoda. Va bene, possiamo pubblicare delle parti della traduzione [N.]

[2:11:41 PM] 1.: pa, ako imamo dva tekstovi koji pricaju na ${ }^{\circ}{ }^{\circ}$ istim nacina ${ }^{\circ}$ mozemo da biti vise objektivne, zar ne?/ Se abbiamo due testi che parlano nello stesso modo possiamo essere più obiettive , ti pare? [N.]

[2:16:06 PM] 1.: da, na tekstu Dragomire spominju i prevod. ali mozemo i analizirati jedan moderni clan* ko prica o juoslevenski pozoriste / Sì, nel testo di Dragomir fanno riferimento anche a una traduzione. Ma possiamo anche analizzare un articolo moderno che parla di teatro jusoglavo [N.]

[2:16:23 PM] J.: aha, misliš da kritičari ${ }^{\circ}$ na različite načine [E.C. ${ }^{\circ}$ pišu o srpskim prestavama i o predstavama koje su prerađene i prevedene na srpski? Aha, pensi che i critici scrivano in modo diverso sulle rappresentazioni serbe e su quelle adattate e tradotte in serbo?

[2:16:28 PM] 1.: Yugo 
[2:17:26 PM] J.: (\#)članakodređeni i neodređeni člani ili član neke stranke, a novinski članak*\#)[E.C]/ L'articolo è determinativo e indeterminativo, il član è anche il membro di un partito, ma il članak è l'articolo di giornale

\section{[2:17:48 PM] J.: članak - određeni i neodređeni član...* [E.C.]}

[2:19:16 PM] 1.: stvarno ne znam. kakve su razlike izmedu njima . Davvero non conosco le differenze tra loro[F.]

[2:19:26 PM] J.: onda moramo naći kritiku neke strane operete,zbog govornih delova, jer u baletu nema govora, pa ni prevoda

Allora possiamo trovare la critica di certe operette straniere, perché ci sono parti parlate, mentre nel balletto non c'è discorso parlato né traduzione [N.]

[2:19:49 PM] J.: misliš da li je član ili članak? Pensi che sia un membro o un articolo? [E.C. + richiesta di disambiguazione e ricerca della comprensione del messaggi del partner]

[2:20:37 PM] 1.: clan i clanak nisu isti rec? Non sono la stessa parola? [I.]

[2:21:21 PM] J.: mogu pogledati da li ima neka takva kritika u kojoj se spominje i prevod drame, mada to onda nema veze sa muzikom i plesom/ Posso dare un'occhiata se per caso non ci siano questo genere di critiche dove si menziona anche la traduzione dei drammi, sebbene non c'entri nulla con la musica e la danza [N.]

[2:24:33 PM] 1.: mislim da nisam razumela. sta znaci članak - određeni i neodređeni član ili član neke stranke, a novinski članak/ Penso che non ho capito davvero cosa significa "članak - određeni i neodređeni član ili član neke stranke, a novinski članak" [I.] ?

[2:24:38 PM] J.: ne. ne kaže se novinski član. Član može da bude kao "određeni i neodređeni član" i član nekog odbora, član stranke (membro)/ No. Non si dice novinski član. Član può essere determinativo o indeterminativo oppure član di qualche organizzazione o di un partiro (membro). [E.C. focus on meaning> disambiguazione del contesto]

[2:24:51 PM] 1.: aaaaa

[2:25:26 PM] 1.: pomislila sam da clan i tekst su sinonima/ Ah, pensavo che fossero sinonimi [F. Conferma comprensione del transfer negativo di tipo lessicale]

[2:25:29 PM] J.: a kaže se novinski članak, ne član :) :)

[2:25:48 PM] 1.: novinski iz novinar, novine [F. Conferma comprensione del transfer negativo di tipo lessicale]

[2:27:27 PM] J.: u italijanskom se "articolo" koristi za pojami član ( u smislu određeni i neodređeni član) i za pojam članak u smislu novinski članak) [E.C. fine del processo di disambiguazione]

$(\ldots)$

Per quanto riguarda invece gli aspetti meta-cognitivi, più strettamente riconducibili alle componenti dell'autonomia dette support e proficiency (cf. grafico I), il feedback formativo del counselor sulla piattaforma può rivelarsi utile per riflettere sui processi in corso.

In questa sede, riportiamo una sintesi alcuni aspetti salienti dell'interazione tra partners e counselors (autori di questo articolo) sul forum wiki:
- La possibilità di esprimersi ciascuno nella propria lingua madre facilita la partecipazione;

- I problemi tecnici legati al software possono trovare una risposta più facile e rapida;

- Lo stimolo a riflettere su "come e cosa stiamo imparando" aiuta ad interpretare meglio il proprio framework di lavoro e a sviluppare metodi di collaborazione più efficaci; 
COUNSELOR: "MI CONFERMATE CHE LA CHAT È PER ORA L'UNICO STRUMENTO AFFIDABILE? MI

SEMBRA DI CAPIRE DI Si” (...)

\section{1) USO DEI CANALI DI COMUNICAZIONE:}

J.: Kao što ste imali prilike da vidite, L. i ja smo komunicirale putem chat-a, a dva puta smo se čule telefonom. Konverzacije sam snimila Call graph-om i pokušala sam da ih stavim na Piattaformu, ali su fajlovi suviše veliki i dugo su se postavljali na sajt. Zbog toga smo odlučile da je bolje da četujemo. Ukoliko postoji neki predlog kako da se ti fajlovi lakše postave na sajt možemo se češće i čuti. Putem chat-a se razmeni manje informacija u odnosu na razgovore, ali ima i dobrih strana jer ostavlja prostora da se razmisli o idejama i objašnjenjima u odnosu na "živ razgovor" Trad. Come avete avuto occasione di osservare, L. ed io abbiamo comunicato via chat e due volte ci siamo sentite via telefono. Le conversazioni le ho registrate con Call graph e ho anche provato a inserirle sulla piattaforma, ma i files sono troppo pesanti e ci mettevano tanto a caricare. Perciò abbiamo deciso fosse meglio chattare. Se esiste un modo di caricarli più velocemente possiamo sentirci più spesso. Attraverso la chat si scambiano meno informazioni rispetto alle conversazioni, ma ci sono anche aspetti positivi perché lascia un maggior spazio per ragionare sulle idee e sulle spiegazioni rispetto alla "viva conversazione".

COUNSELOR: "CERCATE DI NON PERDERE DI VISTA IL FATTO CHE SI FA ETANDEM ANCHE PER "KEEP UP THE LANGUAGE" E DUNQUE L'OBIETTIVO NON È SOLO LA FUTURA PRODUZIONE MICROLINGUISTICA DEL LAVORO CHE FARETE MA ANCHE L'HIC ET NUNC DELLE CONVERSAZIONI" (...)

\section{2) CHAT DIDATTICA:}

J.: Saglasna sam sa tim da smo pomalo zanemarile "forme linguistiche" i fokusirale se na traženje teme za naš rad, ali verujem da ćemo prilikom pisanja rada imati mogućnost da naučimo puno jedna od druge i da unapredimo poznavanje sintakse, morfologije i semantike oba jezika i na taj način obogatimo i naš "akademski jezik". Već nam se dešavalo da odlutamo u "lingvističke teme". Npr. u vezi sa glagolom "scannare" ili imenicom "član" i verujem da će polako biti sve više toga. U svakom slučaju, malo je nezgodno bilo "uplesti se" u puno ispravljanja, jer bismo onda "odlutale" i ne bismo stigle da se fokusiramo na traženje teme. Sada kad smo pronašle temu i kad počnemo da pišemo rad sasvim sigurno će biti toga. Trad. Sono d'accordo col fatto che abbiamo un poco trascurato le forme linguistiche, focalizzandoci sulla ricerca dei temi per il nostro lavoro, ma sono sicura che l'occasione di scrivere insieme un articolo ci darà la possibilità di imparare molto l'una dall'altra e di migliorare la nostra conoscenza sintattica, morfologica e semantica in entrambe le lingue e in tal modo di migliorare il nostro linguaggio accademico. Già ci è successo di soffermarci su temi linguistici come per esempio con il verbo "scannare" (scannerizzare) o con il sostantivo "član"(articolo) e sono convinta che un poco alla volta sarà così sempre di più. In ogni caso creerebbe un poco di disagio soffermarsi troppo sulla correzione, perché finiremmo per disperderci e per perdere il focus sulla ricerca dei temi. Appena avremmo trovato il tema e iniziato a scrivere ci sarà sicuramente spazio anche per quello.

MI SEMBRA DI AVER CAPITO CHE L'IDEA INIZIALE DI SCRIVERE IN TANDEM, L. IN SERBO E J. IN ITALIANO, CON RECIPROCA CORREZIONE E TRADUZIONE FINALE PER UNA VERSIONE BILINGUE SIA STATA IN PARTE ACCANTONATA (...) TENETE PRESENTE CHE SE L'OBIETTIVO È IL RAGGIUNGIMENTO DI UN LIVELLO ACCADEMICO PIÙ ALTO NELLE RISPETTIVE LINGUE OBIETTIVO (SERBO E ITALIANO), ALLORA SARANNO IMPORTANTI ANCHE LE ATTIVITÀ DI PRODUZIONE NON MEDIATE IN MODO COSTANTE DALLA TRADUZIONE DALLA LINGUA MADRE VERSO LA LINGUA OBIETTIVO (...)" 


\section{3) IL NOSTRO PROGETTO:}

J.: Ideja da ja pišem jedan paragraf na italijanskom a L. na srpskom pa da se onda ispravljamo i prevodimo i dalje "stoji", samo što bi jedan deo rada činio prevod srpskih tekstova na italijanski jezik i analiza tih prevoda. Uvod, analizu i zaključak bismo pisale jedan deo na italijanskom (ja), jedan deo na srpskom (L.), pa bismo to ispravljale, pa bismo onda rad prevele na oba jezika. Ne znam da li sam dobro objasnila... L'idea di scrivere io un paragrafo in italiano e L. uno in serbo e di correggerci a vicenda è ancora in piedi. Solo una parte del lavoro sarebbe una traduzione di testi serbi in italiano con relativa analisi. Introduzione, analisi e conclusione le scriveremmo una parte in italiano (io) e una in serbo (L.), per poi correggerle e tradurle in entrambe le lingue. Spero di essermi spiegata bene...

Da quanto è possibile osservare attraverso una lettura dell'estratto di una interazione in forum, le domande poste dal counselor sull'utilizzo della traduzione e sullo stile di correzione all'interno della chat sono dirette a stimolare globalmente i loro processi di metacognizione.

\section{Conclusioni}

Il Teletandem è una modalità di apprendimento che gode di una crescente popolarità, anche grazie alla sempre maggior diffusione dei software ad esso collegati e al loro costante miglioramento.

Un ruolo importante nella popolarizzazione del TT è giocato anche dai numerosi servizi di matching partner. Resta tuttavia aperta la questione di come sviluppare nuovi protocolli, curricolari ed extracurricolari, per l'apprendimento delle lingue in TT. Questa esigenza formativa nasce come effetto della spinta ad integrare il protocollo di base di TT - che si limita a stabilire alcune regole fondamentali di interazione (per es., uso dei canali, numero delle sessioni, strumenti per l'apprendente autonomo, ecc.) - all'interno di un programma di apprendimento di più vasta portata. La metodologia task-based di progettazione, lo sviluppo di ambienti Web 2.0 ad hoc, lo scambio di esperienze maturate sul campo, così come la riflessione sui canali comunicativi nella tele collaborazione (Mullen, Appel, Shanklin, 2009; Belz, 2007; Vinagre, 2007; Lee, 2010; Dooly, 2007) e l'analisi del discorso e della negoziazione dei significati (Pellettieri, 2000, Schwienhorst 2004 ; Tudini, 2005; Liaw-Bunn-Lee Master, 2010) sono ormai temi di ricerca centrali nel panorama complessivo del TT. In questo tipo di contesti di progettazione TT i ruoli degli insegnanti di lingua finiscono sempre giocoforza per essere ridefiniti.

Il counseling nel TT può essere considerato, in generale, come un insieme pianificato di strategie dirette a sostenere bisogno dei partner di stabilire in fase iniziale, (ri)definire e auto-valutare in itinere ed ex post, in modo in(ter)dipendente, i propri obiettivi di studio. Questo bisogno si integra, durante tutto il processo di apprendimento, con la consapevolezza che la messa a fuoco e la realizzazione di tali obiettivi dipende dall'abilità di comunicarli in modo efficace all'interno della coppia e da un'azione regolare e adeguata di reciproco aiuto tra pari.

Nel caso delle microlingue e del nostro esperimento in corso, il counseling potrebbe essere svolto anche da esperti linguistici dello stesso settore o di settori affini, purchè dotati di un certo grado di esperienza pregressa nell'apprendimento in TT. Lo sguardo esterno del counselor, in questo caso, fa parte di quei tipici processi di revisione tra pari all'interno di uno stesso settore professionale, ma acquista un inedito livello di intensità, dal momento che una piattaforma di lavoro come quella illustrata consente un più forte monitoraggio in itinere sulla trattazione dei topics e sullo sviluppo dell'interlingua, in un contesto educativo di interscambio multilinguistico. 


\section{B I B L I O G R A F I A}

- Balboni, P. E. (2000). Le microlingue scientifico-professionali. Torino: UTET.

- Balboni, P. E. (2002). Le sfide di Babele. Torino:UTET.

- Belz, J. A. The Development of Intercultural Communicative Competence in elecollaborative Partnership. In: O' Dowd, R. a cura di (2007). On line Intercultural Exchange. Clevedon: Multilingual Matters.

- Brammerts, H.\&Calvert, M. \&Kleppin, K. Obiettivi e percorsi nella Consulenza individuale. In: Hehmann, G.\&Ponti, D. a cura di (2003). Apprendimento autonomo delle lingue in tandem. Torino: Trauben.

- Brammerts, H., Jonsson, B., Kleppin, K., et al. Consulenza individuale in diversi contesti tandem. In: Hehmann, G., Ponti, D. a cura di (2003). Apprendimento autonomo delle lingue in tandem, Torino: Trauben.

- Cenoz, B. The Effect of Linguistic Distance, L2 Status and Age on Cross-linguistic Influence in Third Language Acquisition. In: Cenoz, B., Hufeisen\& U. Jessner. a cura di (2001). Cross-linguistic Influence in Third Language Acquisition: Psycholinguistic Perspectives. Clevedon: Multilingual Matters

- Cenoz, B. Hufeisen \& U. Jessner a cura di (2001). Cross-linguistic Influence in Third Language Acquisition: Psycholinguistic Perspectives. Clevedon: Multilingual Matters

- Dolci, R. Glottodidattica, costruttivismo e tecnologie. In: Serragiotto, G. a cura di (2004). Le lingue straniere nelle scuole. Torino: Utet Libreria.

- Dunn, W.E. \& Lantolf, J.P. (1998). Vygotsky's Zone of Proximal Development and Krashen's i+1: Incommensurable Constructs; Incommensurable Theories. Language Learning, Vol. 48 No. 3, 411-442.

- Dooly, M. Choosing the Appropriate Communication Tools for an Online Exchange. In: O' Dowd, R. a cura di (2007). On line Intercultural Exchange. Clevedon: Multilingual Matters.

- Guk, L. \& Kellogg, D. (2007). The ZPD and whole class teaching Teacher-led and student-led interactional mediation of tasks. Language Teaching Research, Vol.11 No. 3, 281-299.

- Holec, H. (1981). Autonomy in Foreign Language Learning. Oxford: Pergamon. Lee, L. (2009). Promoting intercultural exchanges with blogs and podcasting: a study of SpanishAmerican telecollaboration. Computer Assisted Language Learning, Vol. 22 No. 5.

- Liaw, M., Bunn-Lee Master, S. (2010). Understanding telecollaboration through an analysis of intercultural discourse. Computer Assisted Language Learning, Vol. 23, No. 1.

- Mullen, T. \&Appel, C.\& Shanklin, T. Skype-based Tandem Language Learning and Web 2.0. In: Thomas, M. a cura di. (2009). Handbook of Research on Web 2.0 and Second Language Learning. Hershey-New York: Information Science Reference.
- Odlin, T. (1989). Language Transfer. Cambridge: CUP.

- Pellettieri, J. Negotiation in cyberspace: The role of chatting in the development of grammatical competence. In: Warschauer,

M., Kern, R. a cura di (2000). Network-based Language Teaching. Cambridge: Cambridge University Press.

- Schwienhorst, K. (2004). Native-Speaker/Non-Native-Speaker Discourse in the MOO: Topic Negotiation and Initiation in a Synchronous Text-Based Environment. Computer Assisted Language Learning, Vol.17 No. 1.

- Telles, A. \& Vassallo, M.L. (2006). Foreign language learning intandem: Teletandem as an alternative proposal in CALLT. The ESPecialist, Vol. 27 No. 2, 189-212.

- Tudini, V. Chatlines for Beginners: Negotiating Conversation at a Distance. In: Holmberg, B., Shelley M., White, C. a cura di. (2005). Distance Education and Languages. Clevedon: Multilingual Matters.

- Vinagre, M. Integrating Tandem Learning in Higher Education. In: O' Dowd, R. a cura di (2007). On line Intercultural Exchange. Clevedon: Multilingual Matters.

- White, C. Autonomy, independence and control: mapping the future of distance language learning. In: Gardner, D., a cura di. (2007). Learner autonomy, Integration and support, Vol. 10. Dublin: Authentik. 


\title{
LANGUAGE FOR SPECIFIC PURPOSES IN THE TELETANDEM ENVIRONMENT, BETWEEN THEORY AND PRACTICE
}

\begin{abstract}
Summary
Teletandem (TT) is as a computer assisted language learning environment in which two students communicate in order to learn each other's native language. In the case of a program focused on Language for Specific Purposes (LSP), TT offers the possibility of putting into contact two people with interests in the same scientific or professional areas, thus configuring a symmetrical relationship which allows mutual help in specialistic areas where access to traditional language teaching may be problematic. In fact, the competence of teachers does not necessarily extend to the highly specialized fields of knowledge mastered by these learners. TT can provide an environment where it is possible to overcome the gap between meaning (which the learner can verbalize in his/her native language) and the linguistic forms required for expression in the target language. The aim of this paper is to present the strengths of TT for LSP and to discuss its critical issues.
\end{abstract}

Key words: language for specific purposes, Teletandem, autonomous learner, counseling, task-based approach. 